\title{
Prognóstico da Estenose Valvar Aórtica Assintomática pelo Teste de Esforço
}

\author{
Marisa Campos Moraes A mato, Paulo Jorge Moffa
}

São Paulo, SP

Objetivo - Avaliar o teste de esforço (TE) como fator preditivo do prognóstico do paciente assintomático com estenose valvar aórtica.

Métodos - Foram acompanhados, por 60 meses, 70 pacientes com área valvar $\leq 1 \mathrm{~cm}^{2}$, sem outra lesão cardíaca, e estudados pelo ecocardiograma Doppler, TE (considerado positivo por alterações eletrocardiográficas ou pela presença de sintomas) e acompanhamento clinico, a fim de ser constatada a morte súbita ou o aparecimento de sintomas.

Resultados - A probabilidade de sobrevida livre de eventos em 16 meses foi de 50\%. Não houve associação, estatisticamente significativa de eventos, com sexo nem com gradiente transvalvar, mas sim, com a idade $<e \geq 50$ anos $(p=0,0124)$, com a área valvar $<e \geq 0,7 \mathrm{~cm}^{2}$ ( $p=0,0003)$ e com a resposta positiva ou negativa do TE $(p=0,0001)$.

Conclusão - Pacientes assintomáticos com estenose importante apresentam baixa sobrevida livre de eventos e o TE é um bom fator preditivo de seu prognóstico.

Palavras-chave: estenose valvar aórtica, teste de esforço, ecocardiografia Doppler

\section{The Value of Exercise Test in the Prognosis of Asymptomatic Patients with Significant Aortic Valve Stenosis}

Purpose - To evaluate the exercise test as a prognostic factor for asymptomatic patients with aortic valve stenosis.

Methods - During 60 months 70 patients with aortic valve area $\leq 1 \mathrm{~cm}^{2}$ without any other heart lesion were studied. The patients underwent Dopplerechocardiograms exercise tests (considered positive in the presence of electrocardiographic changes or symptoms) and clinical follow-up in order to determine the onset of symptoms or sudden death.

Results - The event-free probability was 50\% after 16 months of follow-up. There was no relationship between the occurence of events and the sex or to the transvalvar gradient. However, there was a statistically significant association between the incidence of events ( $p=0.0124)$, valvar area $(p=0.0003)$ and exercise test results $(p=$ 0.0001 ).

Conclusion - Patients with significant aortic stenosis even without symptoms, have a low survival rate and the exercise test is a good prognostic factor during follow-up.

Key-words: aortic valve stenosis, ergometric test, Doppler echocardiography

Arq Bras Cardiol, volume 70 (n 4), 251-255, 1998

Apesar de estarmos vivendo a era da tecnologia e de grande evolução, desde o primeiro implante valvar por Starr Edwards em 1960, ainda não foi encontrado o substituto ideal para as valvas cardíacas. Seja ela metálica ou biológica acarreta sérias complicações; a $1^{\mathrm{a}}$, impõe o uso rotineiro de anticoagulantes e, mesmo assim, podem ocorrer embolias e, a $2^{\mathrm{a}}$, tem pouca durabilidade, levando a várias retrocas.

Instituto do Coração do Hospital das Clínicas - FMUSP

Correspondência: Marisa Campos Moraes Amato - Av. Juriti, 144 - 04520-000 -

São Paulo, SP

Recebido para publicação em 6/11/97

Aceito em 20/1/98
Assim, quanto mais se conseguir preservar uma valva cardíaca doente, menores são as complicações posteriores para o paciente ${ }^{1-9}$. Entretanto, sabe-se que a média de sobrevida com a evolução natural é restrita ${ }^{10-18}$ embora variável, costuma ser de cinco anos, para os portadores de angina, de três anos, para os de síncope e, de dois anos, para aqueles com insuficiência coronária.

Por isso a avaliação para o momento preciso da indicação cirúrgica do paciente com estenose valvar aórtica é muito importante. De um lado, está o risco de morte súbita e, de outro, as complicações das próteses.

A sintomatologia do paciente com estenose valvar 
aórtica é pobre, sendo, muitas vezes, o $1^{\circ}$ evento a morte. Alguns pacientes não dão a devida importância a sintomas esporádicos de tontura e angina e, outros, limitados pela própria rotina da vida cotidiana, realmente não sentem nada.

Assim, tentando objetivar a avaliação clínica desses pacientes, Amato ${ }^{19}$ estudou 69 pacientes com estenose valvar importante e constatou que 59,42\% (41) deles, apesar de estarem assintomáticos, apresentavam sintomas ao se submeterem ao teste de esforço (TE), demonstrando que estavam no limiar da compensação cardíaca.

O objetivo deste trabalho é demonstrar a importância da avaliação dinâmica como fator preditivo do prognóstico de pacientes assintomáticos pela anamnese com estenose valvar importante.

\section{Métodos}

Estudou-se a evolução clínica de 70 pacientes com estenose valvar aórtica importante por período de até 60 meses, sendo 46 (45\%) masculinos, com média de 49,5 (17 a $80, \mathrm{dp}=14,92$ ) anos de idade.

Os critérios para seleção dessa casuística foram: 1) pacientes assintomáticos ou com sintomas não característicos da doença; 2) estenose valvar aórtica importante ou grave ${ }^{20}$ (área de $0,30 \mathrm{a} 1 \mathrm{~cm}^{2}$ ); 3 ) ausência de arritmias ou bloqueio de ramo esquerdo pelo eletrocardiograma (ECG); 4) ausência de coronariopatia ou outras doenças cardíacas comprovadas por cateterismo cardíaco feito, no máximo, seis meses antes do início do protocolo; 5) ausência de hipertensão arterial ou outra doença sistêmica importante.

Os pacientes foram submetidos a ecocardiografia Doppler para o estudo da área valvar e gradiente transvalvar; ao TE e ao acompanhamento clínico por período de até 60 meses.

Foram examinados os pacientes desse protocolo em média a cada três meses repetindo-se o TE a cada seis meses quando o resultado anterior fosse negativo. Identificou-se, pela história clínica criteriosa, a ocorrência de eventos. Considerou-se evento duas possibilidades: a referência de sintomas, como angina, pré-síncope, síncope ou a morte súbita.

Para o ecocardiograma utilizou-se o Aloka SSD 725, 860 e mais recentemente o ATL-Ultramark 9, com transdutor mecânico para o modelo 725, de 3 e 2 megaHertz ( $\mathrm{MHz}$ ) de frequiência, e eletrônico de 3,5 e 2,5 MHz, para o outro. O Doppler pulsátil foi utilizado para localizar o fluxo e medir baixas velocidades e o contínuo, para medir a velocidade máxima.

\begin{tabular}{|cccccc|}
\hline \multicolumn{5}{|c|}{ Tabela I - Caracterização dos pacientes que foram a óbito } \\
\hline Sexo & $\begin{array}{c}\text { Idade } \\
(\text { anos })\end{array}$ & $\begin{array}{c}\text { Área valvar } \\
\left(\mathrm{cm}^{2}\right)\end{array}$ & TE & $\begin{array}{c}\text { Gradiente } \\
(\text { mmHg })\end{array}$ & $\begin{array}{r}\text { Evolução } \\
(\text { meses })\end{array}$ \\
\hline M & 41 & 0,38 & + angina & 110 & 4 \\
F & 59 & 0,60 & + angina & 80 & 16 \\
M & 48 & 0,48 & + angina & 99 & 5 \\
M & 49 & 0,49 & + Y e $\Delta P$ & 136 & 6 \\
\hline
\end{tabular}

\begin{tabular}{|c|c|c|c|c|}
\hline \multirow{2}{*}{ Variável } & \multicolumn{2}{|c|}{ Com evento (38) } & \multicolumn{2}{|c|}{ Sem evento (32) } \\
\hline & $\mathrm{n}$ & $\%$ & $\mathrm{n}$ & $\%$ \\
\hline Sexo masculino & 26 & 68,42 & 20 & 62,50 \\
\hline Idade $<50$ anos & 16 & 42,10 & 25 & 78,12 \\
\hline$\geq 50$ anos & 22 & 57,89 & 7 & 21,87 \\
\hline Área valvar $<0,7 \mathrm{~cm}^{2}$ & 32 & 84,21 & 11 & 34,37 \\
\hline$\geq 0,7 \mathrm{~cm}^{2}$ & 6 & 15,78 & 21 & 65,62 \\
\hline Gradiente $\leq 70 \mathrm{mmHg}$ & 13 & 52,00 & 16 & 50,00 \\
\hline$>70 \mathrm{mmHg}$ & 25 & 65,78 & 15 & 46,87 \\
\hline Teste de esforço positivo & 34 & 89,47 & 9 & 28,12 \\
\hline Teste de esforço negativo & 4 & 10,52 & 23 & 71,87 \\
\hline Teste de esforço positivo - sintoma & 21 & 55,26 & 2 & 6,25 \\
\hline Teste de esforço positivo - outro & 13 & 34,21 & 7 & 21,87 \\
\hline
\end{tabular}

A técnica usada permitiu o estudo do gradiente e a determinação da área do orifício valvar aórtico. O gradiente utilizado para este estudo foi o gradiente instantâneo máximo medido em mmHg. Para seu cálculo foi necessário o alinhamento paralelo entre o fluxo e o feixe ultra-sônico, porque a determinação da velocidade está relacionada com o co-seno do ângulo formado entre essas duas variáveis. Este cálculo já vem numa planilha do equipamento e para obter valor preciso é necessário que a localização da estenose esteja correta.

O cálculo da área do orifício valvar aórtico foi feito pela equação de continuidade ${ }^{21-25}$, medido $\mathrm{em}^{2} \mathrm{~cm}^{2}$, que estabelece que, quando o fluxo dentro do sistema vascular (Q1) iguala ao fluxo fora do sistema (Q2), o produto da velocidade (V1) pela área de corte seccional (A1), no $1^{\circ}$ ponto, iguala o produto da velocidade (V2) pela área (A2), no $2^{\circ}$ ponto. Por um rearranjo da equação é evidente que a área de corte seccional no local da estenose (A2) pode ser calculada $\operatorname{como}(\mathrm{A} 2)=(\mathrm{A} 1) \mathrm{x}(\mathrm{V} 1) /(\mathrm{V} 2)$.

Para o TE empregou-se como ergômetro, a esteira rolante de rampa móvel, que dispensa, na maioria das vezes, treinamento prévio, pois os movimentos sobre ela são os que normalmente se executam na deambulação. Os aparelhos utilizados foram: o sistema Fukuda Denshi, ML -8000 e Mat 2100 esteira rolante e, da Funbec, monitor 4-1TC/FC, esteira rolante e eletrocardiógrafo ECG-4.

Utilizou-se o protocolo de Ellestad ${ }^{26}$. Foram feitas aferições periódicas da $\mathrm{PA}$ e da freqüência cardíaca $(\mathrm{FC})$, associadas com a visibilização contínua do ECG interrompendo-

\begin{tabular}{|llrr|}
\hline \multicolumn{3}{|c|}{ Tabela III - Resultado do teste de esforço } \\
\hline Resultado Teste de esforço & Motivo associado & $\mathrm{n}$ & $\%$ \\
\hline \multirow{2}{*}{ Positivo } & Sintomas e Y e $\Delta$ P & 3 & 4,3 \\
& Sintomas e Y & 3 & 4,3 \\
& Sintomas e $\Delta$ P & 10 & 14,3 \\
& Sintomas apenas & 7 & 10,0 \\
& Y e P & 9 & 12,9 \\
& Y apenas & 8 & 11,4 \\
& $\Delta$ P apenas & 2 & 4,3 \\
\multirow{2}{*}{ Negativo } & & 27 & 38,6 \\
\hline \multirow{2}{*}{ Total } & & 70 & 100,0 \\
\hline
\end{tabular}




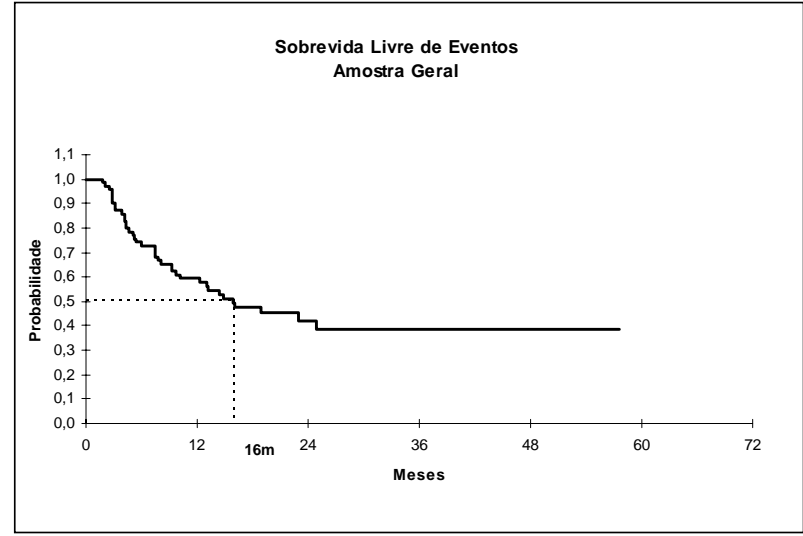

Fig. 1 - Curva atuarial demonstrando a probabilidade de sobrevida livre de eventos até 60 meses.

se dentro das condições normais, quando o indivíduo tivesse atingido a FC denominada máxima ou submáxima. A FC máxima é calculada através da fórmula FC máxima $=210$ (idade $\mathrm{x} 0,65$ ) e a submáxima corresponde a $85 \%$ desta. Em vista de eventuais complicações decorrentes da realização desse exame, todo o cuidado foi tomado em relação ao local onde o mesmo foi realizado, aos equipamentos e medicamentos necessários.

Considerou-se positivo os testes com: a) modificações eletrocardiográficas, como: 1) infradesnivelamento do segmento ST, de morfologia horizontal e/ou descendente com ponto $\mathrm{Y}$ (medido a $80 \mathrm{~ms}$ do ponto $\mathrm{J}$ ) sendo $\geq 1 \mathrm{~mm}$, para o homem e $\geq 2 \mathrm{~mm}$ para a mulher; 2) infradesnivelamento do segmento $\mathrm{ST}$ ascendente com ponto $\mathrm{Y} \geq 2$ para o homem e $\geq 3$ para a mulher. b) Sem alteração eletrocardiográfica e interrompidos por: 1) angina; 2) síncope; 3) arritmias ventriculares ou 4) manutenção ou aumento da PA ( $\mathrm{P}=$ diferença da PA sistólica em repouso e durante o exercício) $<20 \mathrm{mmHg}$ com o incremento do trabalho. Adotou-se o critério estabelecido pela American Heart Association ${ }^{27}$ para interrupção do teste.

A análise estatística foi feita através do método de Kaplan Meyer ${ }^{28}$ para construção das curvas demonstrando a estimativa da probabilidade de sobrevida livre de eventos. Para a comparação das curvas empregou-se o método de Log-Rank.

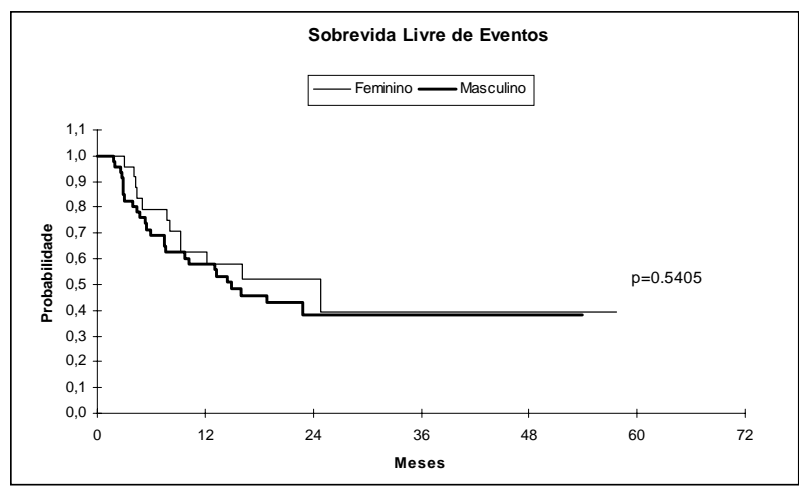

Fig. 2 - Curvas atuariais mostrando que o sexo não influencia na incidência de eventos.

\section{Resultados}

O acompanhamento clínico dos pacientes, durante 60 meses mostra, conforme a curva de sobrevida livre de eventos (fig. 1), a probabilidade de $50 \%$ de um paciente com estenose valvar aórtica importante estar livre de eventos após 16 meses do diagnóstico. A tabela I caracteriza os pacientes que foram a óbito.

A ocorrência de eventos foi relacionada com as variáveis: sexo, idade, área valvar, gradiente transvalvar, resultado positivo ou negativo do TE e TE positivo com aparecimento de sintomas (tab. II).

A análise pela curva de sobrevida livre de eventos de-

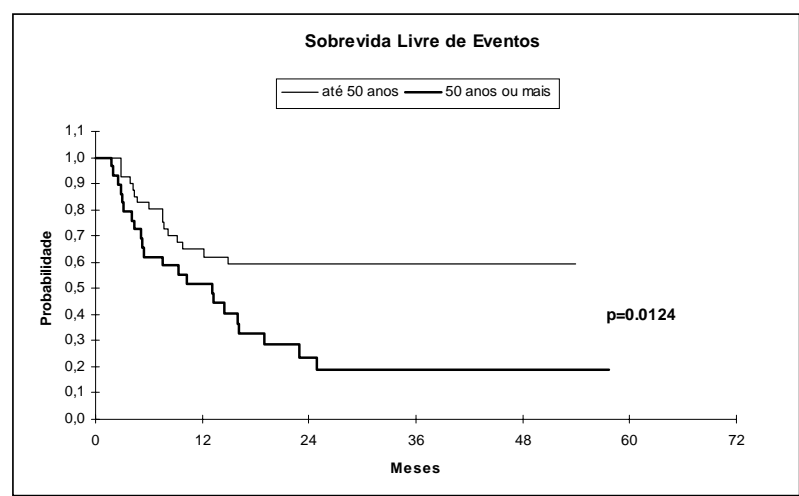

Fig. 3 - Curvas atuariais mostrando a influência significativa da idade na sobrevida.

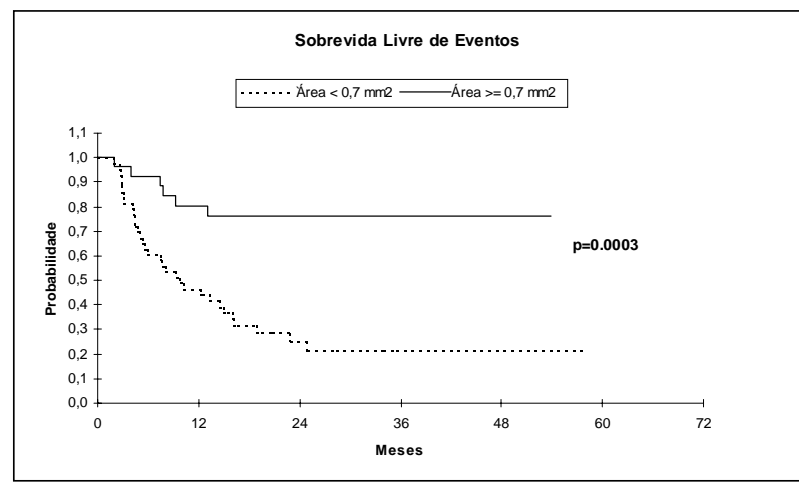

Fig. 4 - Curvas atuariais mostrando a maior incidência de eventos em pacientes com área valvar $<0,7 \mathrm{~cm}^{2}$

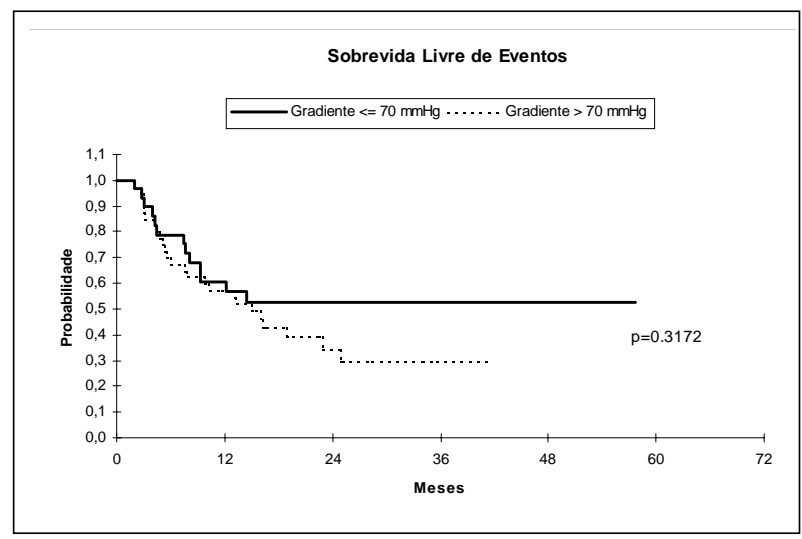

Fig. 5 - Curvas atuariais mostrando que o gradiente transvalvar praticamente não modifica a curva de sobrevida. 


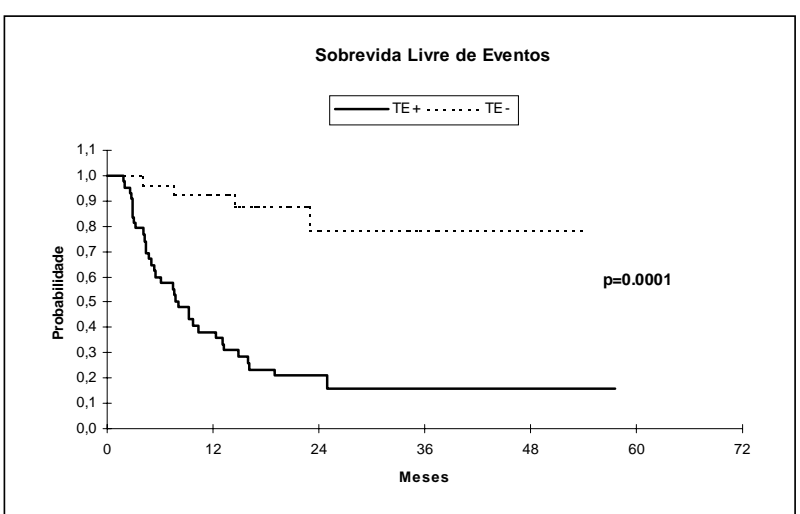

Fig. 6 - Curva atuarial mostrando a diferença estatisticamente significativa da incidência de eventos em pacientes com teste de esforço positivo e negativo.

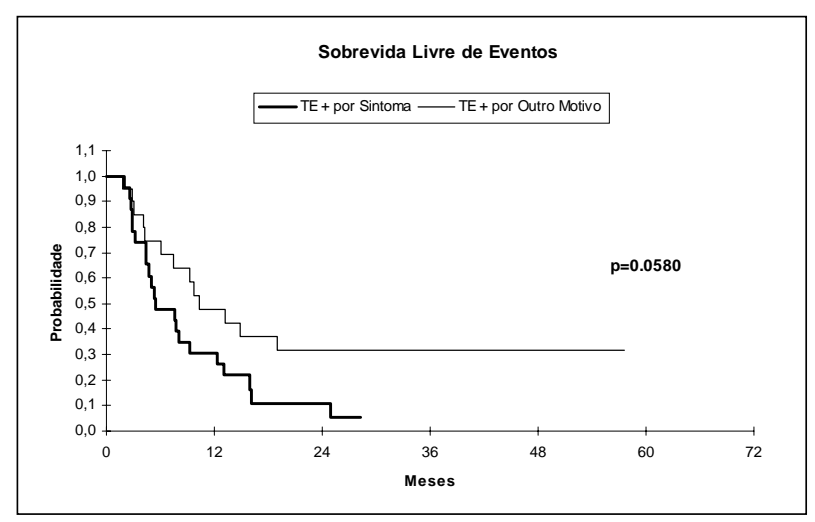

Fig. 7 - Curva atuarial mostrando a maior incidência de eventos em pacientes com teste de esforço positivo por sintomas.

monstrou que não houve diferença estatisticamente significativa na incidência de eventos em cada sexo $\mathrm{p}=0,5404$ (fig. 2).

A curva de sobrevida livre de eventos apresentou comportamento diferente para o grupo de pacientes com idade $<e \geq 50$ anos ( $p=0,0124)$, (fig. 3).

A incidência de eventos foi maior no grupo de pacientes com área $<0,7 \mathrm{~cm}^{2}$, sendo a diferença estatisticamente significante com $\mathrm{p}=0,0003$, (fig. 4 ).

A incidência de eventos nos grupos de pacientes com gradiente $\leq \mathrm{e}>70 \mathrm{mmHg}$ não são estatisticamente diferentes $\mathrm{p}=0,3172$, conforme as respectivas curvas de sobrevida na figura 5 .

O TE foi positivo em $43(61,4 \%)$ pacientes. Os determinantes deste resultado estão relacionados na tabela III .

A incidência de eventos ocorreu em 38 pacientes, sendo que $34(89,47 \%)$ tinham TE positivo, e apenas $4(10,52 \%)$ tinham TE negativo, sendo esta diferença estatisticamente significativa com $\mathrm{p}=0,0001$.

As curvas de sobrevida dos grupos de pacientes com TE positivo e negativo mostram comportamento estatisticamente diferentes ( $\mathrm{p}=0,0001)$, (fig. 6).

Os pacientes que apresentaram TE positivo pela presença de sintomas 21/34 tiveram incidência maior de eventos durante sua evolução. Entretanto não houve diferenças estatisticamente significativas entre os eventos deste grupo e do grupo com TE positivo por outras causas. Este dado ratifica a importância discriminatória do teste positivo seguindo os critérios adotados. A figura 7 mostra as curvas de sobrevida livre de eventos de pacientes com TE positivo por sintoma ou por outro motivo.

\section{Discussão}

A avaliação dinâmica completando o estudo hemodinâmico pelo cateterismo cardíaco, para os valvopatas, é utilizada desde $1970^{29-37}$, sendo posteriormente introduzida em associação ao estudo radioisotópico ${ }^{38} \mathrm{e}$, mais recentemente, também utilizada, isoladamente ${ }^{19}$.

Na história natural da estenose valvar aórtica há longo período de latência, durante o qual ocorre aumento gradual da estenose, sem manifestação de sintomas.

Vários autores ${ }^{38-40}$ não aceitam a idéia de serem operados doentes assintomáticos e procrastinam a operação até a manifestação de sintomas, enquanto outros ${ }^{14,41,42}$ apóiamse em parâmetros hemodinâmicos, considerando o gradiente transvalvar e, mais recentemente, a área do orifício aórtico, os elementos indicativos para a decisão cirúrgica.

Embora o quadro clínico seja sempre soberano, o fato da sintomatologia poder estar mascarada pela inatividade ou autolimitação do paciente, valoriza o esforço, interagindo com esses exames.

Este trabalho mostra, pela análise atuarial, a probabilidade de 50\% de sobrevida livre de eventos em 16 meses, demonstrando que, apesar de assintomáticos, esses pacientes apresentam alta morbidade e mortalidade.

O gradiente transvalvar de $70 \mathrm{mmHg}$, estabelecido na literatura ${ }^{14,41}$, como parâmetro hemodinâmico de gravidade da lesão, dividiu dois grupos de pacientes que apresentaram curva de sobrevida livre de eventos, semelhantes, sem diferenças estatísticas, mostrando, assim, ser um parâmetro não muito confiável para estabelecer o prognóstico dessa doença. Tal fato ocorre, possivelmente, por ele variar em função do débito cardíaco, fração de ejeção que, por sua vez, variam em função da sobrecarga do ventrículo esquerdo, do estado de contratilidade do miocárdio e da resistência arterial sistêmica ${ }^{40}$.

O menor tamanho da área valvar, associado à maior incidência de eventos $(84,21 \%$ dos pacientes com área < que $0,7 \mathrm{~cm}^{2}$ apresentaram eventos) mostra que o grau de estenose, por si só, é um parâmetro importante para avaliar o prognóstico desses pacientes. Entretanto, no decorrer deste estudo, alguns pacientes com orifício menor que esse valor não apresentaram eventos, enquanto outros com valor maior, apresentaram eventos em dois anos (fig. 4).

Observou-se também que, dos quatro óbitos, três apresentavam área valvar $<0,5 \mathrm{~cm}^{2}$ e todos TE positivo (tab. I).

As curvas atuariais dos grupos de pacientes com TE positivo e negativo tiveram comportamento estatisticamente diferente, demonstrando a importância desse exame como fator preditivo do prognóstico desses pacientes.

Assim, o TE, associado à medida da área valvar, pelo ecodopplercardiograma, melhora a avaliação do paciente assintomático pela anamnese com estenose valvar aórtica, 
podendo selecionar, mais criteriosamente, os pacientes que ainda possuem reserva miocárdica e podem praticar determinada atividade física, daqueles que necessitam de correção cirúrgica, mesmo que seja para o implante de um substituto valvar. Pacientes com estenose valvar aórtica importante, mesmo que assintomáticos, apresentam baixa sobrevida livre de eventos.

A incidência de eventos é significativamente maior em pacientes com área valvar $<0,7 \mathrm{~cm}^{2}(84,21 \%)$ do que naqueles com área entre $0,7 \mathrm{~cm}^{2} \mathrm{e} 1 \mathrm{~cm}^{2}(15,78 \%)$.

A incidência de eventos em pacientes com TE positivo é significativamente maior $(89,47 \%)$ do que naqueles com teste negativo $(10,52 \%)$.

OTE mostrou-se de grande valor na avaliação do prognóstico do paciente assintomático com estenose valvar aórtica importante.

\section{Referências}

1. Giuliano ER - Cardiac protheses: a twentieth century problem. Int J Cardiol 1983; 3: 203-5.

2. CohnL-The long-term results of aortic valve replacement. Chest 1984; 85:387-96.

3. Gallo I, Ruiz B, Duran CG - Isolated mitral valve replacement with the hancack porcine bioprothesis in rheumatic heart disease: analysis of 213 operative survive followed up 4.5 to 8.5 years. Am J Cardiol 1984; 53: 178-81.

4. Magilligan DJ, Lewis Jr JW, Titley B, Peterson E - The porcine bioprothetic valve. J Thorac Cardiovasc Surg 1985; 89: 499-507.

5. Hartz RS, Fisher E, Fuikelmeier B et al - A light year experience with porcine bioprosthetic cardiac valve. J Thorac Cardiovasc Surg 1986; 91: 910-17.

6. Pomerantzeff PMA, Abreu MCS, Amato MCM et al - Retroca valvular. Rev Bras Cirur Cardiovasc 1987; 2: 180-8.

7. Amato MCM, Pomerantzeff F, Grinberg M et al - Implante de prótese de Starr Edwards há mais de 15 anos: análise de 34 casos. Arq Bras Cardiol 1987; 48: 299 301.

8. Amato MCM, Pomerantzeff F, Grinberg M, Belotti G, Pileggi F - Evolução imediata e tardia de pacientes com prótese porcina. Arq Bras Cardiol 1988; 51 : 381-4.

9. Cormier B, Luxereau P, Bloch C et al - Prognosis and long-term results of surgically treated aortic stenosis. Eur Heart J 1988; 9 (suppl.): 113-20.

10. Olesen KH, Warburg E - Isolated aortic stenosis: the late prognosis. Acta Med Scand 1958; 160: 437-46.

11. Ross J, Braunwald E - Aortic stenosis. Circulation 1968; 37: 61-7.

12. Cohen LS, Friedman WF, Braunwald E-Natural history of mild congenital aortic stenosis elucidated by serial hemodynamic studies. Am J Cardiol 1972; 30: 1-5.

13. Frank S, Johnson A, Ross JJ - Natural history of valvular aortic stenosis. Br Heart J 1973; 35: 41-6.

14. Rapaport E - Natural history of aortic and mitral valves disease. Am J Cardiol $1975 ; 35: 221-7$

15. Turina J, Hess D, Sepulcri F, Krayenbuehl HP - Spontaneous course of aortic valve disease. Eur Heart J 1987; 8: 471-83.

16. Nitta M, Nakamura T, Hultgren HN, Bilisoly J, Marques B - Noninvasive evaluation of the severity of aortic stenosis in adults. Chest 1987; 91: 682-7.

17. Nitta M, Nakamura T, Hultgren HN, Bilisoly J, Tovey DA-Progression of aortic stenosis in adult men. Chest 1987; 92: 40-3.

18. Pellikka PA, Nishimura RA, Bailey KR, Tajik AJ - The natural history of adults with asymptomatic, hemodynamically significant aortic stenosis. J Am Coll Cardiol 1990; 15: 1012-7.

19. Amato MCM - Estenose aórtica assintomática: avaliação pelo teste de esforço. Acta Cir Bras 1995; 10: 77-83.

20. Rahimtoola SH-Perspective on valvular heart disease: update II. In: Era in Cardiovascular Medicine. New York: Elsevier, 1991: 45-70.

21. Seitz WS - Cardiac valve area formula for the assessment of aortic stenosis based upon evaluation of left ventricular wall stress in diastole. Int J Cardiol 1985; 9: 225-30.

22. Odemuyira O, Hall RJC - Assessing the severity of valve stenosis. Br Heart J 1986; 55: 117-9.

23. Richards KL, Cannon SR, Miller JF, Crawford MH - Calculation of aortic valve area by Doppler echocardiography: a direct application of the continuity equation. Circulation 1986; 73: 964-9.

24. Smith MD, Kwan OL, Demarta AN - Value and limitations of continuous-wave Doppler echocardiography in estimating severity of valvular stenosis. Jama 1986; 255: 3145-51

25. Come PC, Riley MF, Ferguson JF, Morgan JP, Mckay RG - Prediction of severity of aortic stenosis: accuracy of multiple noninvasive parameters. Am J Med 1988; 85: 29-37.

26. Ellestad MH - Stress Testing: Principles and Practice. 1975.

27. Anderson FL, Tsagaris TJ, Tikoff G, Thorne JL, Schmidt AM, Kuida H-Hemodynamic effects of exercise in-patients with aortic stenosis. Am J Med 1969; 46: 872-85.

28. Lee EP - Statistical Methods for Survival Data Analysis.: Life Time Learning Publication, 1980: 75-154.

29. Lee SJK, Jonsson B, Bevergard S, Karlof I, Astrom H, - Hemodynamic changes at rest and during exercise in-patients with aortic stenosis of varying severity. Am Heart J 1970; 79: 318-31.

30. Friedman WF, Modlinger J, Morgan JR - Serial hemodynamic observations in asymptomatic children with valvar aortic stenosis. Circulation 1971; 43: 91-7.

31. Cueto L, Moller JH-Haemodynarnics of exercise in children with isolated aortic valvular disease. Br Heart J 1973; 35: 93-8.

32. Richardson JW, Anderson FL, Tsagaris TJ - Rest and exercise hemodynamic study in-patients with isolated aortic stenosis. Cardiology 1979; 64: 1-11.

33. Nestico PF, Depace NL, Kimbiris D et al - Progression of isolated aortic stenosis: analysis of 29 patients having more than 1 cardiac catheterization. Am J Cardiol 1983; 52: 1054-8.

34. Osbakken MD, Bove AA - Use of left ventricular filling and ejection patterns in assessing severity of chronic mitral and aortic regurgitation. Am J Cardiol 1984; 53: $1054-60$.

35. Nylander E, Ekman I, Marklund T, Sinnerstad B, Karlsson E, Wranne B - Severe aortic stenosis in elderly patients. Br Heart J 1986; 55: 480-7.

36. Wisenbaugh T, Elion JL, Nissen SE-Influence of aortic valve disease on systolic stiffness of the human left ventricular myocardium. Circulation 1987; 75:964-72.

37. Iskandrian AS - Valvular heart disease. In: Myron CG - Cardiac Nuclear Medicine. New York: McGraw-Hill, 1987: 371-94.

38. Iskandrian AS, Heo J - Radionuclide angiographic evaluation of left ventricular performance at rest and dining exercise in patients with aortic regurgitation. Am Heart J 1986; 111: 1143-9.

39. Kelly TA, Rothbart RM, Cooper M, Kaiser DS, Smucker ML, Gibson RS - Comparison of outcome of asymptomatic to symptomatic patients older than 20 years of age with valvular aortic stenosis. Am J Cardiol 1988; 61: 123-30.

40. Braunwald E-On the natural history of severe aortic stenosis. J Am Coll Cardiol 1990; 15: 1018-20.

41. Horstkotte DD, Loogen F - The natural history of aortic valve stenosis. Eur Heart J 1988; 9: 57-64.

42. Griffith MJ, Carey C, Coltart DJ, Jenkins BS, Webbeploe MM - Inaccuracies in using aortic valve gradients alone to grade severity of aortic stenosis. Br Heart J 1989; $62: 372-8$ 